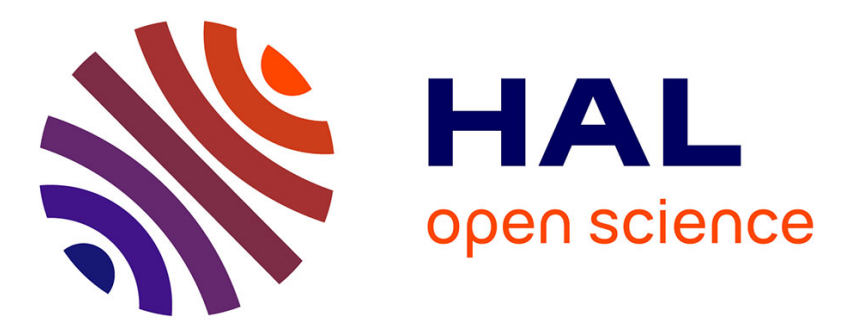

\title{
A digital-enabled framework for intelligent collaboration in small teams
}

\author{
Juanqiong Gou, Qinghua Liu, Wenxin Mu, Wenchi Ying, Hamideh \\ Afsarmanesh, Frederick Benaben
}

\section{- To cite this version:}

Juanqiong Gou, Qinghua Liu, Wenxin Mu, Wenchi Ying, Hamideh Afsarmanesh, et al.. A digitalenabled framework for intelligent collaboration in small teams. 20th Working Conference on Virtual Enterprises (PRO-VE), Sep 2019, Turin, Italy. pp.193-202, 10.1007/978-3-030-28464-0_18 . hal02290873

\section{HAL Id: hal-02290873 \\ https://imt-mines-albi.hal.science/hal-02290873}

Submitted on 14 Feb 2020

HAL is a multi-disciplinary open access archive for the deposit and dissemination of scientific research documents, whether they are published or not. The documents may come from teaching and research institutions in France or abroad, or from public or private research centers.
L'archive ouverte pluridisciplinaire HAL, est destinée au dépôt et à la diffusion de documents scientifiques de niveau recherche, publiés ou non, émanant des établissements d'enseignement et de recherche français ou étrangers, des laboratoires publics ou privés. 


\title{
A Digital-enabled Framework for Intelligent Collaboration in Small Teams
}

\author{
Juanqiong Gou ${ }^{1,1}$, Qinghua Liu ${ }^{1}$, Wenxin $\mathrm{Mu}^{1}$, Wenchi Ying ${ }^{1}$, \\ Hamideh Afsaramanesh ${ }^{2}$ and Frederick Benaben ${ }^{3}$ \\ ${ }^{1}$ School of Economic and Management, Beijing Jiaotong University, Beijing, China \\ \{jqgou,18120615,wxmu,wcying\}@bjtu.edu.cn \\ ${ }^{2}$ Institute of Informatics, University of Amsterdam, Netherlands \\ h.afsarmanesh@uva.nl \\ ${ }^{3}$ École des Mines d'Albi-Carmaux, France \\ frederick.benaben@mines-albi.fr
}

\begin{abstract}
Socialized value creation in networked organizations is the new paradigm of digital organizations. In dynamic and uncertain environment, small teams and big organizations can benefit from interaction with intelligent assistance. For that purpose, various behavior and relationships in situations should be effectively collected and fused. This paper proposes very early steps toward developing theoretical method for the intelligent collaboration of small teams and their big organization environment. Three main subjects are addressed in this paper: (i) Behavior intelligence support for virtual organizations; (ii) Situations behavior digitization, focusing on scenario recognition and modeling; (iii) Data fusion and behavior pattern mining, based on situation-based behavior modeling and heterogeneous behavior model fusion.
\end{abstract}

Keywords: Intelligent collaboration, Collaborative Networks, Digital platform, Situations

\section{Introduction}

In big data environment, value creation in a social and networked way driven by realtime market insight [1] is a new trend. It also provides a new paradigm, which information system field strives to establish and analyze. For this, establishment of organizational network ecosystems and its collaboration mechanism provide the core foundation.

There are two trends defined in organizational ecological evolution [1]. One is driven by business model innovation, in which more external organizations and users are included in the value creation system. The other trend, which we will concentrate more in this paper, is to let employees participate in value creation and value

The present research has been supported by Research on Behavior Intelligence in High Education Environment Based on Collaborative Scenarios (2018A02008). 
distribution. In this way, internal boundaries of organizations are being weakened, and new organizational structures are emerging to replace the traditional "company + employees" structure which driven by its leaders. Among them, "big organization + small teams" is a well-recognized organizational structure [2]. Here, "big organization" doesn't mean organizations which is pretty huge, but a concept corresponding with "small teams", acting as a platform to support mall teams with internal and external resources. In this structure, small teams can be established rapidly to make agile response to emerged opportunity, and the big organization can support small teams with internal and external resources. However, in existing organization management, small teams cannot make effective interactions with the big organization during their life cycles, which makes it hard to fully exploit the advantages in this organizational structure [3]. Therefore, between the collaboration in small teams and the overall resources organizing in the big organization, a systemic contradiction appears. This makes it challenging to perform unified modeling and operation management in a single environment.

Referred to the theory of Collaborative Networks [4], this study aims to provide theoretical methods for intelligent collaboration of small teams in big organization environment with high-level design to realize the new vision.

The paper is structured as follows. Section 2 gives an overview of existing contributions that help to solve the mentioned tasks. Section 3 presents a framework aimed at providing a theoretical method for intelligent collaboration in small teams in a big organization environment. Section 4 details the early steps on three main subjects to achieve a theoretical vision for this research in the previous section by digital platforms. Finally, section 5 presents the conclusion.

\section{Related Works}

\subsection{Collaborative Networks}

New organization forms need theoretical models to formalize them. The paradigm of Collaborative Networks $(\mathrm{CN})$ provides a new perspective for solving the challenge of small teams' collaboration in big organizations [4]. Collaborative Networks address reconstructing organizations into two high levels of Collaborative Networked Organizations (CNO) namely: the goal-oriented networks and longer-term strategic alliances [5].

Virtual organization is a typical form of goal-oriented networks. Compared with the general understanding of the concept of virtual organization in the field of organizational management, the theory of Collaborative Networks emphasizes the dynamism of $\mathrm{VO}$, which requires VOs themselves to be either driven by continuous production / service provision activities, or driven by the goal of grasping a single collaboration opportunity [5].

Longer-term strategic alliance, mostly represented as a VO Breeding Environment (VBE), is an association or pool of organizations and their related supporting 
institutions [6]. Members in a VBE have both the potential and the will to share resources and cooperate with each other through the establishment of a "base" longterm cooperation agreement. When a business opportunity is identified by one member of VBE that acts as a broker, a subset of these organizations will be selected to form a VO, in order to fulfill the opportunity. A breeding environment, as a longterm networked structure, provides in advance the adequate base environment for the establishment of the cooperation agreements, the provision of common co-working and co-development infrastructures, generating the common ontologies, and establishing mutual trust as the main aspects, which are necessary to facilitate building a new $\mathrm{VO}$ [7]. For small teams in a big organization environment, the theory of $\mathrm{CN}$ addresses the needed digital models, prototype systems and key technical methods.

With the continuous growth and dynamic evolvement of CNOs, it is necessary for VOs to preserve and enhance its digital integration with VBE. In other words, it is necessary to establish the collaborative mechanism for VO-VBE at two levels: (i) to drive VBEs gathering information dynamically, realizing the intelligent service to support VOs, and (ii) to help digital management of VOs and their information feedback to VBEs.

\subsection{Situations and Artificial Intelligence}

Artificial intelligence (AI) is "designed to realize human intelligence by machine means" [8]. Since the late 1970s, AI has shown great promise in improving human decision-making processes in various business endeavors due to its ability to recognize business patterns, learn business phenomena, seek information, and analyses data intelligently [9]. In our research, we aim to provide small teams varies kinds of service based on data assets of their big organization environment. These services will help small teams just like extra partners, giving them proper information at proper time, so that small teams can collaborate "intelligently".

A situation can be defined as "a snapshot of a complete world state at a particular time" [10]. It is "the missing link" to organize and fuse diverse data resources for computational intelligence in software development [11]. Big data brings artificial intelligence into situation applications [12] and brings fundamental changes to the computing architecture and application models [3]. In information systems, collaboration in small teams mainly relies on situation applications, and its behavior data is integrated with the enterprise legacy system to form enterprise data assets. However, how to build a digital collaborative platform based on data assets and make full use of artificial intelligence is still a problem remained to be studied. Since it can help to better solve the organizational collaboration challenges between the small teams their big organization environment, solving this problem constitutes the core entry point of our research. 


\section{Overview of Objectives}

In traditional management models, an enterprise has vertical boundaries (departments) and horizontal boundaries(levels) dividing its members and resources into small "cells". While in theory of Collaborative Networks, VOs can be created from a VBE triggered by a specific business/collaboration opportunity. One of the main ideas of our research is to apply VO-VBE model into a single enterprise environment. In this way, a VBE doesn't act as a long-term association of autonomous organizations, but a collection of members and resources within a single enterprise. This shows the following advantage: the potential of creating goaloriented, dynamic organization models replaces the traditional static model, which in turn makes it possible for enterprises to make quick reactions toward emerging opportunities and act as a "big organization" from which "small teams" can be produced continuously.

Actually, the VO-VBE model not only provides a new view for organization itself, but also creates new views for businesses, resources and data assets. Based on this, our research fuses these views into the VO-VBE model and proposes a comprehensive mechanism for small teams/big organizations, while supporting highfrequency interactions to fully exploit their advantages.

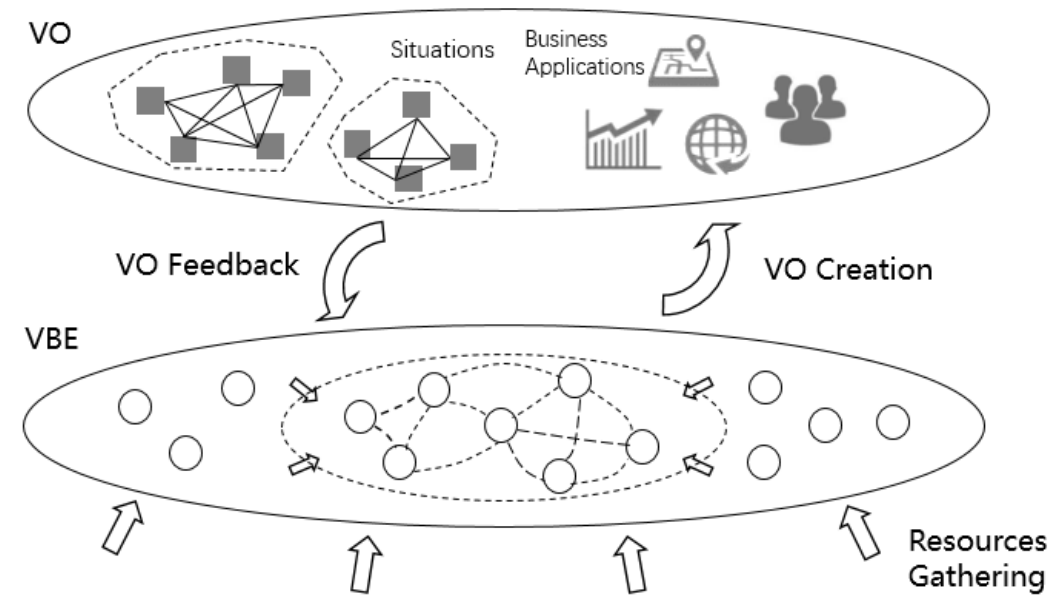

Fig. 1. Digital-enabled VO-VBE collaboration framework

As is shown in Figure 1, a VBE can be assumed as a pool of a wide variety of resources within a big organization. Each small circle in the VBE pool in Figure 1 is a unit containing some kinds of resources, such as a machine, space or other infrastructure. A human, such as an expert with specific skills, or a partnership with external organizations can also be resources needed to support VOs.

There are two kinds of arrows around and inside the VBE resources pool. One represents all kinds of resources being gathered from the "open universe" of external resources. In this process, administrators or other agents of the VBE bring these nodes 
and resources into the boundary of the VBE. In other words, VBE makes all resources ready and prepared for potential participation [13], while this will include making some agreements or preparing complete profiles of resources, etc. With this process, which will be further explained below, the VBE provides a sound base for VO creation, no matter which building situations or business applications emerge.

The other arrows inside the VBE pool, however, represent the process of building strong connections between members of resources. The VO-VBE model describes a dynamic collaboration mechanism, and that means assignment of resources to collaboration is also dynamic. For example, after a period of collaboration in a VO, members in the VO gain more experience and knowledge, and will have a better understanding of their partners, skills and infrastructures. These new experiences, knowledge and understanding, can be conveyed back to VBE as new connections, and make relevant members and resources "more prepared" to collaborate for the next times. This process is shown in Figure 1 as "VO Feedback" and arrows inside the VBE pool pointing from single circles to connected circles.

While the VBE in this model concentrates mainly on resources, the VO layer focuses more on business. As VOs in theory of Collaborative Networks are highly goal-oriented and dynamic, their goals and business can be modeled as several situations, or a chain of situations. Situation models contain possible elements required to describe certain business [14]. They can be built to fit newly emerged opportunities and can also come from predesigned situations from the VBE resources pool. Based on situations which carry all the business information, a final model for business applications becomes clear. In other words, which members and resources are relevant, what kinds of information need to communicate and how to communicate, what processes are needed and what is the purpose of each process etc. The situation-based business applications also make it possible to provide VO members with various kinds of AI service. As we will explain in the next section, during the whole lifecycle of VOs, the situations, business applications, behavior data and all other kinds of connections are feeding back to the VBE. This will make the big organization better prepared for the subsequent planned small teams' collaborations.

\section{High-Level Design of Proposed Framework}

In order to realize the required theoretical framework described above, in our proposed model, three components must be developed. The following main aspects are shown in Figure 2: (i) Behavior intelligence support for VOs, addressing the VO situation applications and their intelligent processing; (ii) situations digitization, addressing steps involved in situation modeling and VO creation; (iii) data fusion and behavior pattern mining, addressing steps to realize a VBE resources pool. In the next three subsections, we describe each of these components with more details. So far, the proof-of-concept about framework in 3 is in progress. We are proceeding our work cooperating with two Chinese software companies on these three components. 


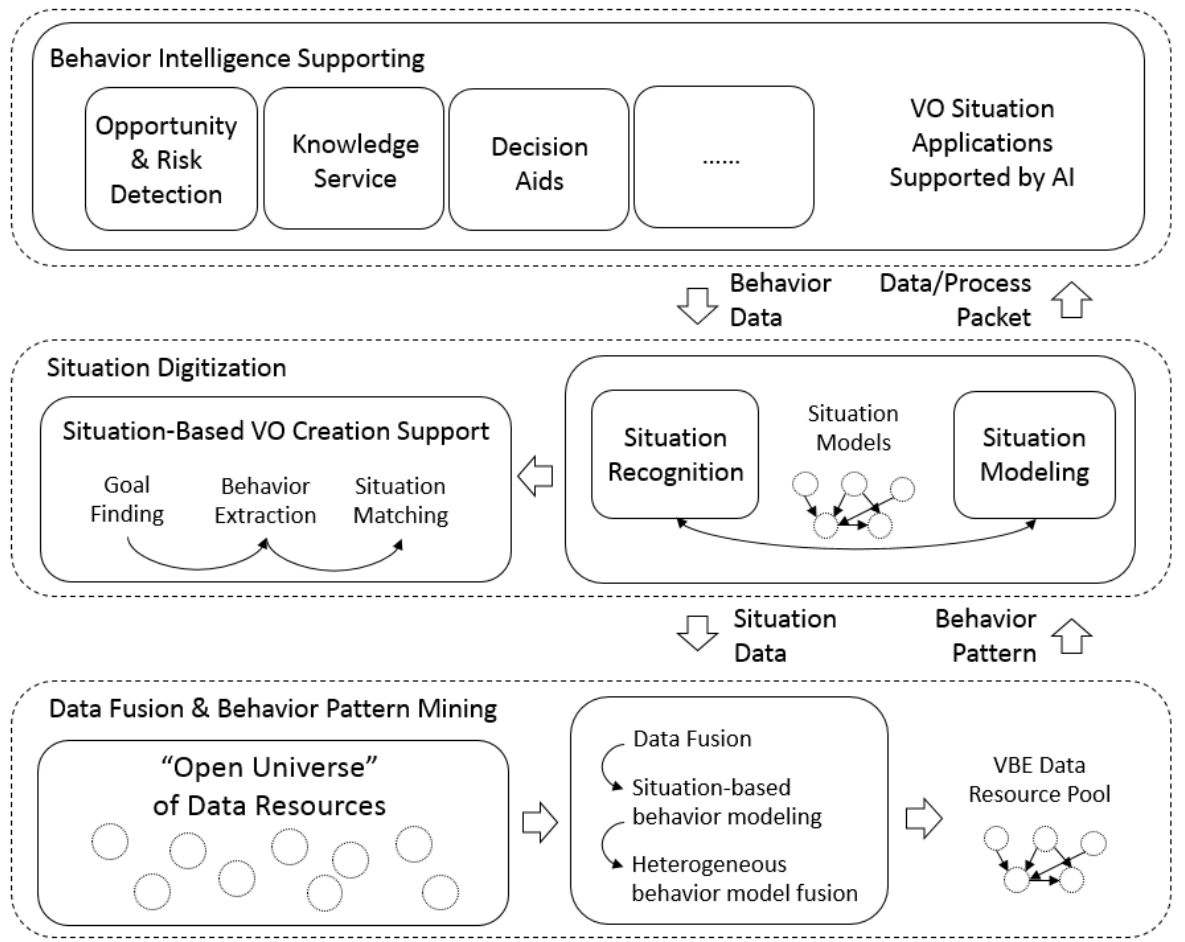

Fig. 2. High-level design of proposed framework

To make this high-level design more concrete, we suppose that there is a regional manufacturing network, acting as a "big organization" or VBE. Mike is a broker of this network mainly in charge of marketing affairs. One day, Mike got a requirement to do a market research for a new product. On the digital platform, this task was recomposed into several situations automatically: team creation, research planning, research data collection, data analysis, etc.

\subsection{Behavior Intelligence Supporting for VOs}

This component focuses on various kinds of VO situation applications, such as opportunity/risk detection, knowledge service, decision aids etc. Realization of these behavior intelligence applications will be considered as the means to provide an ideal vision for small teams' collaboration in the future. This vision needs to be based on a well-implemented VBE digital platform, and just as it was described above, behavior data from all running applications will be fed back to situation digitization and finally make a close loop with the whole VBE digital platform.

In the example of Mike and his market research, the situation applications in team creation phase may include team composition and partner recommendation. The VBE digital platform can help Mike find appropriate partners based on their past behaviors in the same or other situations. The behavior data may imply that a potential partner 
has related experience before so that AI can recommend him or her to Mike. At the same time, behaviors in this situation will also be recorded, such as what kind of partners does Mike prefer or what ability is needed in this kind of task, to help collaboration in other small teams in the future.

In the information system for the small team and its big organization, the situation application will receive the data packets and process packets, and the following is planned: firstly, the data packet will be parsed to form a visual interface guided by the situation behavior, which is the visualization of the situation participants' behavior mode; secondly, the process packet is parsed to form the situation application with behavior mode's visual interface.

Incorporating AI in its development, this structure assists VO decision-making and behavior guidance by identifying dynamic opportunities and risks in situations, thus enhancing intelligent operation for small teams.

\subsection{Situation Digitization}

To realize the VO situation applications that we mentioned in section 4.1, situations need to be recognized and represented from reality to information systems, and we call this part of works situation digitization. This component focuses on the collection of information from processes of situations, design of the required situation models, definition and representation of related behaviors, and implementation of the collection and storage of data for these aspects. Actually, this part of the research can be regarded the connection between $\mathrm{VO}$ and VBE.

Situation recognition. There are two sources to get information for situation recognition, thus laying the foundation for the construction of the specific situation model. The first source is the demands, which mainly include the observation method or the interview method. Getting situation recognition information from demands requires observation and investigation of the key participants in the situations, so that the activities of the participants in a certain period can be sorted out and concisely defined, and then the specific situations will be summarized. The second source is the collected data. Gathering situation information from data mainly requires analyzing the data generated during the interaction of the participants. The data can be either structured data or unstructured data. Then for different characteristics of the data, proper analysis and mining methods will be applied to summarize important information related to the situation (such as the situation mode, key processes involved in the situation, etc.) as they are needed to provide a basis for the classification of situations. In our market research example, most situations have already been recognized or defined from demand by knowledge and experience from experts. But if data showing that Mike and his team did much behaviors not suit for existing situation mode, the AI of the digital platform will decide whether to model a new situation.

Situation modeling. Situation model in our research bases on the design of metamodel for collaborative situations in [15], which mainly include context, partners, 
objectives and behavior. Elements and relationships between elements will be described, and we will use ontology or UML to represent them. The collaborative situations will be manually classified according to the collected situation information. Here, the situations are mainly classified into two types: process-based situations and interactive situations. Process-based situations are service-oriented, and their aim is to transfer information or resources from one entity to another, such as data collection or data analysis of Mike's market research. This kind of situations is built around the process and includes multiple sets of processes and collaboration between processes. Interactive situations are based on frequent interactions, such as instant messaging, face-to-face brainstorming etc. Thus, the information generated in this kind of situations is potentially dynamic and unstructured. Appropriate modeling methods will be selected according to different situation types, but both types are based on the basic structure of the metamodel.

Situation-based VO creation. When the required situation models are established, the subsequent tasks include identifying, defining, and describing the behaviors for the VO, and determining the virtual organization network that can achieve the collaborative goals. Here, we first find and refine the collaborative goals, such as finding proper partners, collecting available research data. Second, extract behavior or the events and activities related to the behavior in the situation model, and then determine the key behavior or activities, which should be the activities that can be further subdivided into participant behavior according to the requirements of the behavior collection. Finally, the collaborative goals will be matched with the situation behavior, and the participants and resources will be selected from the VBE resource pool to complete the creation of the VO. In other words, this step is to match each situation with relevant participants and to find out what kind of behaviors they will do and what processes and resources are needed to complete the goals.

\subsection{Data Fusion and Behavior Pattern Mining}

This component mainly focuses on realizing ideas about VBE resource pool which were mentioned in section 3. Its main functionality includes situation-based behavior modeling and data fusion. The component will involve developing effective data sharing mechanisms in VBE. Further, data collection, data cleaning, and data fusion technologies will be developed to implement data management for related behavior pattern discovery and mining. Following are the four main aspects to be supported by this component.

Data fusion. Since the interactive behavior data in situations or other kinds of data describing resources is mostly unstructured text, such as online message between members in market research team. Here the data must be pre-processed and then fused. The unstructured or semi-structured data should be dimension-reduced, and then the heterogeneous data is realized through pattern alignment and instance alignment. The application of an appropriate data sharing mechanism and an enhanced VBE resources pool for data will be established. This is also a prerequisite for the following aspects. 
Situation-based behavior modeling. We use appropriate modeling methods such as the OWL primitives to construct multi-dimensional situation ontology, in order to complete the description of basic behavior. Second, in each situation, we select the appropriate machine learning algorithm to effectively analyze behavior of data and realize data-driven behavior model. Finally, in each situation, the behavior models obtained by knowledge-driven and data-driven methods are encoded and stored.

Heterogeneous behavior model fusion. Data fusion methods are divided into incremental fusion and full-scale fusion. Since in practice it is not possible to obtain all data models once, our research proposes an incremental heterogeneous data model fusion method, which is mainly divided into three steps: (i) data parsing, (ii) pattern alignment, and (iii) instance alignment.

\section{Conclusion}

The main topic addressed in this paper is how to enhance collaborative evolvement for small teams and their big organization environment on digital platforms, giving small teams more intelligent assistance and promoting frequent interactions to fulfill their goals in a dynamic environment.

Virtual organization - VO, and virtual organizations breeding environment - VBE, are two typical organizational models introduced for Collaborative Networks. We propose a VO-VBE two-layer model to provide a possible perspective to solve systemic differences and sometimes even contradictions between the business goals and the operation mechanism applied to small teams and their big organizations. As big data brings artificial intelligence into the situation application, situations show a perspective that can help organizations to fulfill digital collaboration with artificial intelligence.

Based on existing research, this paper proposes the high-level framework for intelligent collaboration in small teams in their big organization environment. This framework fuses several state-of-the-art views, through which the VBE layer concentrates mainly on resources and the VO layer focuses on business. VBE will then give strong support for VO creation by resources and situations, while during the whole lifecycle of VOs all kinds of connections and situation behavior information will be fed back to the VBE to enhance next collaboration.

The final realization of the framework needs to be based on developing effective information systems and tools. This paper addresses three main components of works to achieve the goals for this framework: (i) behavior intelligence support provides the base to achieve intelligent VO operation; (ii) situations digitization can be regarded as a connection between VO and VBE to complete a dynamic link of resources and situations; finally, (iii) data fusion and behavior pattern mining focuses on realizing VBE resources pool, including an effective data sharing mechanism and the implementation of data collection for related behavior pattern discovery and mining. 
To sum up, several key issues for the social value creation and network of small teams are addressed at the high level in this paper. Next steps of our work address the development and final establishment of organizational network ecosystem and collaboration mechanisms supporting small teams.

\section{References}

1. Feng, Z., Guo, X., Zeng, D., Chen, Y. \& Chen, G. On the research frontiers of business management in the context of Big Data. Journal of Management Sciences in China 16, (2013).

2. Xiao, J., Mao, Y. \& Xie, K. Transformation of Chinese Manufacturing Industry: Insight from Intelligent Manufacturing System Based on Internet and Big Data. Modern Industrial Economy (2), 5-16 (2016).

3. Gou, J., Li, N., Mu, W., Liu, Q. \& Lv, X. Chinese Collaborative Software in Digital Transformation Era. in COLLABORATIVE NETWORKS OF COGNITIVE SYSTEMS 534, 601-611 (SPRINGER INTERNATIONAL PUBLISHING AG, 2018).

4. Camarinha-Matos, L. \& Afsarmanesh, H. Collaborative networks: a new scientific discipline. Journal of Intelligent Manufacturing 16, 439-452 (2005).

5. Camarinha-Matos, L. M. \& Afsarmanesh, H. Classes of collaborative networks. in of Networked \& Virtual Organizations 193-198, Idea Group (2008).

6. Afsarmanesh, H., Camarinha-Matos, L. M. \& Msanjila, S. S. Models, Methodologies, and Tools Supporting Establishment and Management of Second-Generation VBEs. IEEE Transactions on Systems, Man and Cybernetics - C, 41(5): 692-710. (2011).

7. Afsarmanesh, H. \& Camarinha-Matos, L. M. A framework for management of virtual organizations breeding environments. In: Collaborative Networks and their Breeding Environments, Springer, pp. 35-48, Valencia, Spain, 26-28 Sep 2005, (2005).

8. Cai Shushan;Xue Xiaodi;Wu Lingwe; Artificial Intelligence and Human IntelligenceOn Human-Computer Competition from the Five-Level Theory of Cognitive Science. Contemporary Social Sciences 140-155 (2017).

9. Min, H. Artificial intelligence in supply chain management: theory and applications. International Journal of Logistics Research \& Applications 13, 13-39 (2010).

10.Magoutas, B., Mentzas, G. \& Apostolou, D. Proactive Situation Management in the Future Internet: The Case of the Smart Power Grid. in International Workshop on Database \& Expert Systems Applications (2012).

11.Chang, C. K. Situation analytics - at the dawn of a new software engineering paradigm. Science China Information Sciences 61, 050101 (2018).

12.Banuls, V. A., Turoff, M. \& Roxanne Hiltz, S. Collaborative scenario modeling in emergency management through cross-impact. TECHNOLOGICAL FORECASTING AND SOCIAL CHANGE 80, 1756-1774 (2013).

13.Afsarmanesh, H., Camarinha-Matos, L. M. \& Ermilova, E. VBE Reference Framework. In: Methods and Tools for Collaborative Networked Organizations, 35-68, Springer (2008)

14.Bidoux, L., Bénaben, F. \& Pignon, J. P. A Metamodel for Collaboration Formalization. (2014).

15.Benaben, F.; Li, J.; Gou, J. A tentative framework for risk and opportunity detection in a collaborative environment based on data interpretation. In: HICSS 2019, DOI:10.24251/hicss.2019.369 (2019). 